\title{
Lifesaving Treatment of Aortic Valve Staphylococcus aureus Endocarditis: Daptomycin and Early Surgical Therapy
}

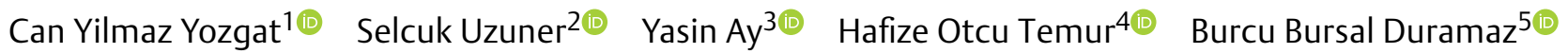

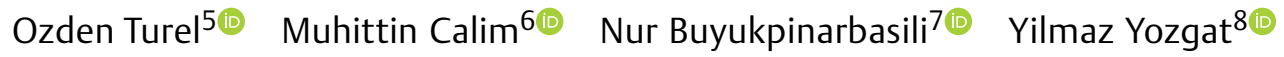 \\ 1 Faculty of Medicine, Bezmialem Vakif University, Istanbul, Turkey \\ 2 Department of Pediatric Critical Care Medicine, Bezmialem Vakif \\ University, Istanbul, Turkey \\ ${ }^{3}$ Department of Cardiovascular Surgery, Bezmialem Vakif University, \\ Istanbul, Turkey \\ ${ }^{4}$ Department of Radiology, Bezmialem Vakif University, Istanbul, Turkey \\ ${ }^{5}$ Department of Pediatric Infectious Diseases, Bezmialem Vakif \\ University, Istanbul, Turkey \\ ${ }^{6}$ Department of Anesthesiology and Reanimation, Bezmialem Vakif \\ University, Istanbul, Turkey \\ ${ }^{7}$ Department of Pathology, Kanuni Sultan Suleyman Training and \\ Research Hospital, Istanbul, Turkey \\ ${ }^{8}$ Department of Pediatric Cardiology, Bezmialem Vakif University, \\ Istanbul, Turkey \\ Address for correspondence Can Yilmaz Yozgat, MD, Faculty of \\ Medicine, Adnan Menderes Bulvari, Vatan Caddesi, Bezmialem Vakif \\ University, 34093 Istanbul, Turkey \\ (e-mail: yozgatyilmaz@gmail.com). \\ J Pediatr Intensive Care 2021;10:298-302.
}

\author{
Abstract \\ Keywords \\ - infective endocarditis \\ - Staphylococcus aureus \\ - open cardiac surgery \\ - daptomycin \\ - pediatric intensive \\ care unit
}

Infective endocarditis (IE) is an uncommon infection in children. The recommended treatment for native valve endocarditis secondary to methicillin-susceptible Staphylococcus aureus infection is antistaphylococcal penicillins such as nafcillin or oxacillin. If the initial therapy fails in IE, it can lead to catastrophic results. Nowadays, daptomycin is the best alternative antimicrobial agent to treat children with severe infections, when standard antimicrobial therapy does not yield a result. Herein, in this article, we described a case of a 16-year-old boy who had aortic valve $S$. aureus endocarditis with septic embolization and stroke. The patient was successfully treated only with daptomycin as well as surgical therapy in the early phase of the infection.

\section{Introduction}

Infective endocarditis (IE) is an uncommon infection in children. The estimated annual incidence is 0.3 to 0.8 per 100,000 per year in children older than 1 year and adolescents. ${ }^{1}$ Congenital heart disease (CHD), central venous catheters, and rheumatic heart disease (RHD) are major risk factors for developing IE. Among 36 children diagnosed as definite or probable IE between the years 2004 and 2011 in Turkey, CHD was present in $61.1 \%{ }^{2}$ The most frequently isolated organisms were Streptococcus viridans, followed by Staphylococcus aureus and Staphylococcus epidermidis.

received

March 11, 2020

accepted

May 13,2020

published online

July 30,2020

The recommended treatment for native valve methicillinsusceptible S. aureus (MSSA) endocarditis is antistaphylococcal penicillin, either nafcillin or oxacillin. ${ }^{1}$ Daptomycin is a cyclic lipopeptide that has been approved by the U.S. Food and Drug Administration (FDA) to be used in the treatment of patients older than 18 years with endocarditis due to $S$. aureus. Daptomycin is an excellent way to treat children with rightsided endocarditis, especially when standard antimicrobial therapy does not yield a result. Herein, we describe a case of a 16 -year-old boy who had aortic valve $S$. aureus endocarditis with septic embolization and stroke. The patient was

(c) 2020. Thieme. All rights reserved. Georg Thieme Verlag KG,

Rüdigerstraße 14,

70469 Stuttgart, Germany
DOI https://doi.org/ 10.1055/s-0040-1713614. ISSN 2146-4618. 
successfully treated with only daptomycin as well as surgical therapy in the early phase of the infection.

\section{Case Report}

A 16-year-old boy presented with a medical history of severe headache and drowsiness to the emergency department (ED). Physical examination in the ED revealed a high fever of $39.5^{\circ} \mathrm{C}$, heart rate of 90 beats per minute, respiratory rate of 20 breaths per minute, and blood pressure of $140 / 90 \mathrm{~mm} \mathrm{Hg}$. After physical examination in the ED, the initial decision was to refer the patient to the departments of pediatric cardiology and pediatric nephrology due to his ongoing high blood pressure. A transthoracic echocardiographic (TTE) examination revealed mild insufficiency of the aortic valve ( - Video 1 ), although three cusps of the aortic valve were observed. Therefore, C-reactive protein (CRP), antistreptolysin $\mathrm{O}$, and throat culture of the patient were checked to see if the patient might have the condition of acute rheumatic fever (ARF). The initial blood result of the patient was normal, and rapid antigen group A Streptococcus test was negative. His medical history was significant for recurrent tonsillitis and migratory polyarthralgia. The patient's condition of aortic insufficiency was attributed to RHD. Therefore, he was started on intramuscular (IM) benzathine penicillin of 1.2 million units for a 21day basis. The Department of Pediatric Nephrology was also consulted for his ongoing high blood pressure. His renal examination was unremarkable. Thus, the patient was sent back home on analgesic therapy.

\section{Video 1}

Transthoracic echocardiography showing mild aortic insufficiency of the aortic valve. Online content including video sequences viewable at: https://www. thieme-connect.com/products/ejournals/html/ 10.1055-s-0040-1713614.

A day later, the patient developed a high-grade fever, severe headache, and somnolence. Again, the patient was admitted to the ED. In his second admission to the ED, a few septic petechiae were noted on his soles, palms, and especially on his index finger (- Fig. 1). Additionally, petechiae were also detected on the patient's foot ( - Fig. 2). TTE showed moderate insufficiency of the aorta and also revealed a new-onset trivial mitral regurgitation compared with the examination which had been done before the second admission to the ED. We also performed transesophageal echocardiography (TEE) to detect the existing vegetation in the aortic root and mitral valve. However, we did not detect any vegetation in the heart. Even though we could not see any vegetation, IE was diagnosed due to clinical manifestations. Daptomycin of $8 \mathrm{mg} / \mathrm{kg} / \mathrm{d}$ was promptly initiated after obtaining two sets of blood cultures from two different veins. At the end of 24 hours of the second admis-

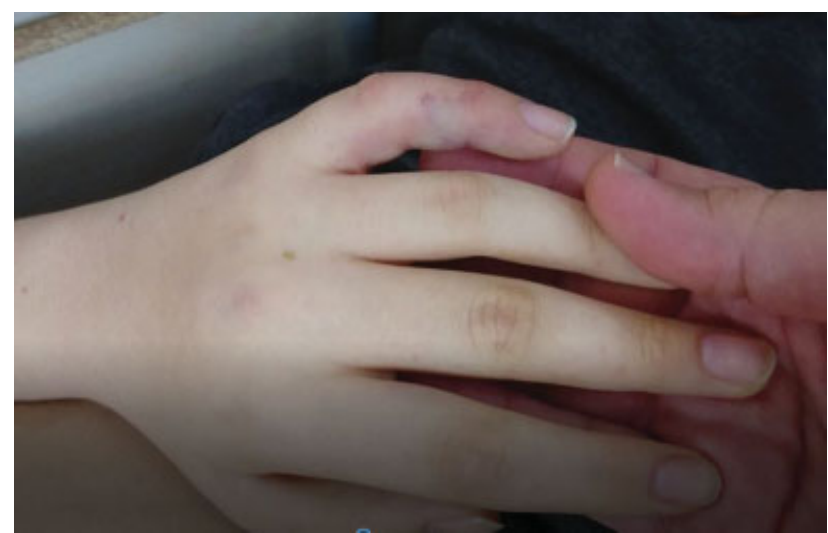

Fig. 1 Figure showing septic petechiae on sole and palms and especially on the index finger.

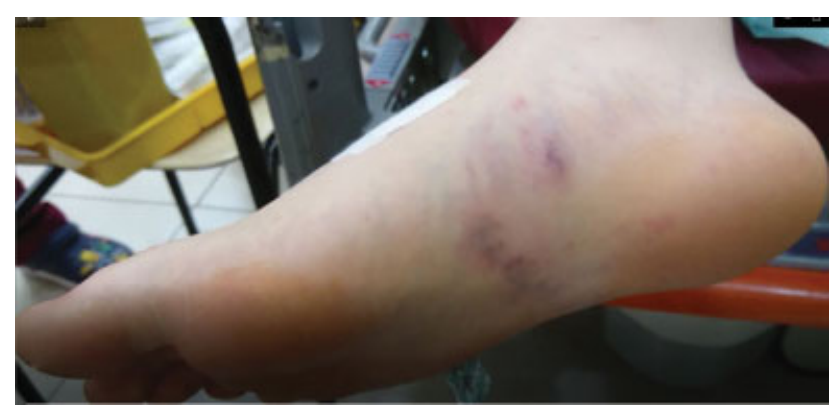

Fig. 2 Figure showing septic petechiae on the foot.

sion to the ED, his neurologic condition was deteriorated severely. There was a significant increase in the number of petechiae throughout the patient's extremities. Also, cranial computed tomography revealed several cerebral emboli in the nucleus caudate of the patient's brain. Thus, the patient was transferred to our institution's pediatric intensive care unit (PICU) for a higher level of care.

Bilevel positive airway pressure (BiPAP) was started immediately upon PICU admission. Despite 24 hours of BiPAP support, the patient remained unchanged. Therefore, the patient was intubated. Of note, his oxygen saturation was $98 \%$. Pentoxifylline was promptly initiated for the circulatory compromise of his lower extremities. Laboratory investigations showed hemoglobin of $11 \mathrm{~g} / \mathrm{dL}$, white blood cells of $22 \times 109 / \mu \mathrm{L}$, renal function (blood urea $40 \mathrm{mg} / \mathrm{dL}$, serum creatinine $0.8 \mathrm{mg} / \mathrm{dL}$ ), CRP of $21 \mathrm{mg} / \mathrm{L}$, and high erythrocyte sedimentation rate of $65 \mathrm{~mm} / \mathrm{h}$. The first sets of cardiac enzymes were troponin-I $3.5 \mathrm{IU} / \mathrm{mL}$, creatine kinase $(\mathrm{CK})$ $210 \mathrm{IU} / \mathrm{mL}, \mathrm{CK}-\mathrm{MB}$ isoenzyme $16 \mathrm{IU} / \mathrm{L}$, amylase $185 \mathrm{~g} / \mathrm{dL}$, and albumin $3.78 \mathrm{~g} / \mathrm{dL}$. On the second day of his PICU admission, the patient's case was discussed in a council which was composed of two pediatric cardiologists, a pediatric heart surgeon, two pediatric infectious disease specialists, and two pediatric critical care specialists. A decision was reached to proceed with a surgical operation immediately for several reasons: the extreme deterioration of the cerebral condition of the patient, the rapid spread of septic emboli throughout the patient's extremities and his brain, and the existing left heart IE. 


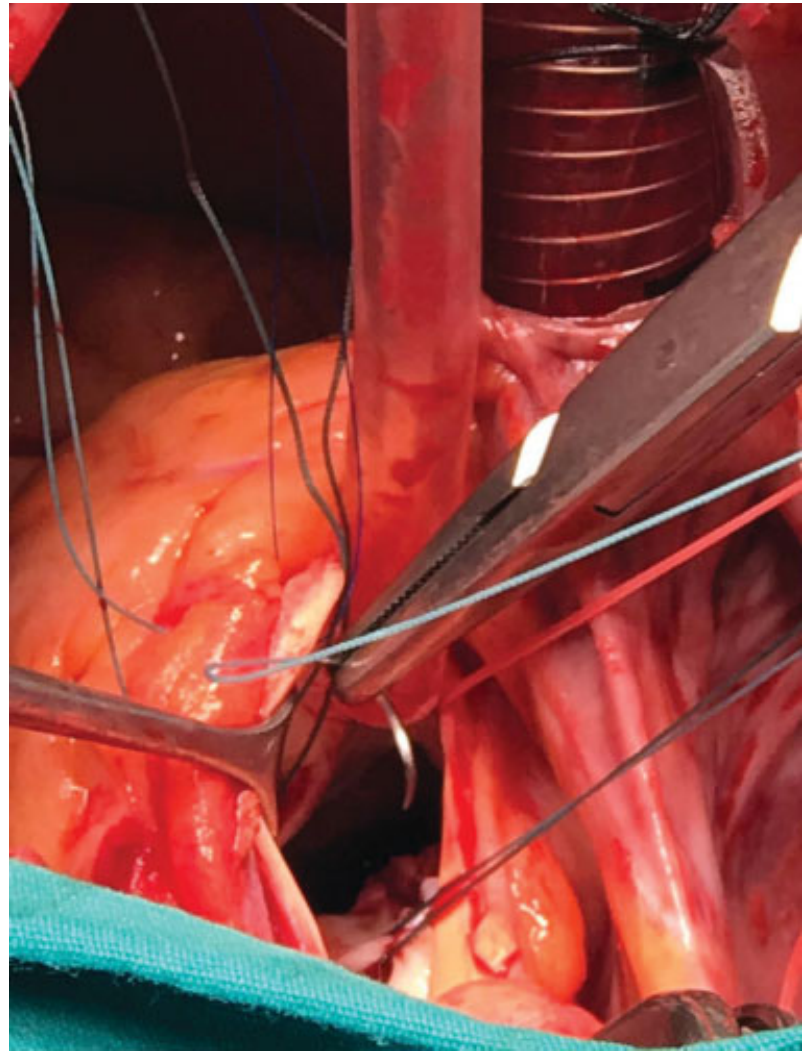

Fig. 3 Operative image illustrating the large vegetation on the left ventricular outflow tract beneath the aortic valve.

Before the start of open cardiac surgery with aortic valve replacement, a repeat TEE was done by an anesthesiologist. There was suspicious finding of the vegetation, measuring $\sim 1 \times 1.2 \mathrm{~cm}$ in diameter, in the left ventricular outflow tract (LVOT). Surgery was performed to the patient on his third day of PICU admission. Aortic valve exploration was performed by oblique aortotomy. There was an erosion in the noncoronary cusp, and there were significant fibrotic alterations in other valves. The chordae were also noted to be thickened. A vegetation, $\sim 2 \times 1.5 \mathrm{~cm}$ in diameter, was detected. The vegetation was located on the ventricular side of the aortic annulus, and it extended through the LVOT (-Fig. 3). The surgeon concluded that the deformations in aortic valves occurred due to the patient's chronic rheumatic fever. Aortic

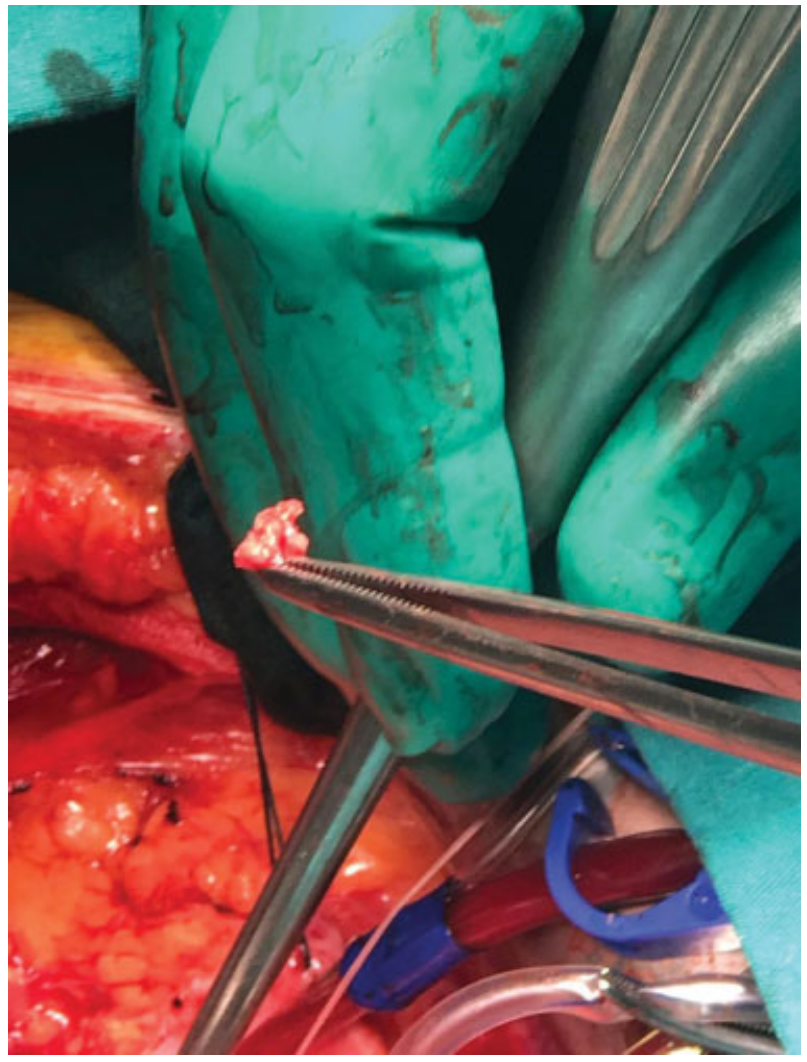

Fig. 4 Operative image illustrating the large vegetation and surrounding infected tissues.

valve and vegetations were resected, and no abscesses were detected (-Fig. 4). A \#21 St. Jude Medical Regent mechanical heart valve was implanted in the aortic position. The resected vegetation was sent to pathology department for further analysis. The histopathological analysis of the vegetation revealed the existence of fibrin exudation and polymorphonuclear leukocyte communities on the surface of the valve tissue (-Fig. 5A, 5B). Hyalinization and fibrosis were observed all over the valve ( $\mathbf{- F i g} \mathbf{5 C}$ ). Thus, in addition to the findings which were presented on the surface, this view was compatible with chronicity of ARF.

Initial blood cultures grew MSSA. Only daptomycin therapy was continued after the surgery. The postoperative clinical condition of the patient improved significantly, fever

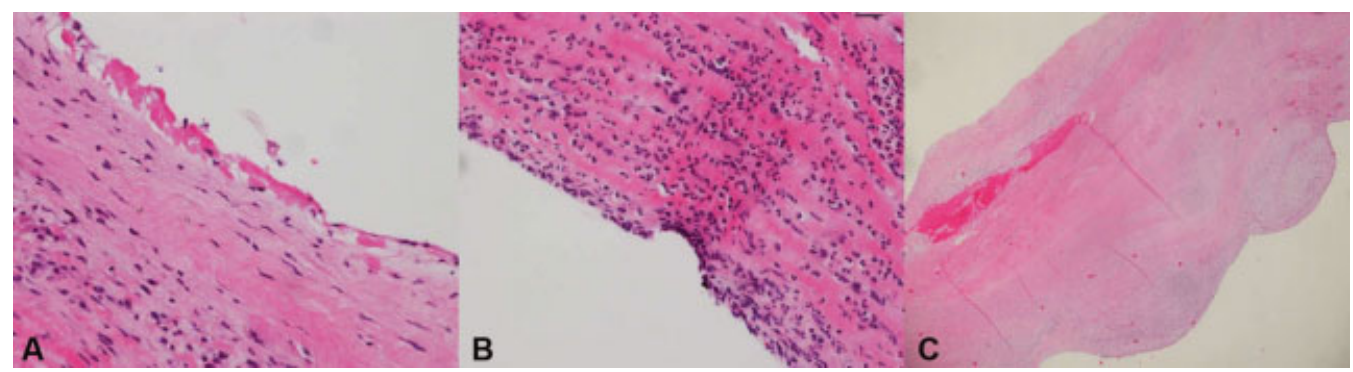

Fig. 5 (A) Histopathological image showing the existing of fibrin exudation and polymorph nucleus leukocyte communities on the surface of the valve tissue $(\mathrm{H}-\mathrm{E} \times 200)$. (B) Histopathological image showing polymorph nucleus leukocyte communities on the surface of the valve tissue (H-E $\times 200)$. (C) Histopathological image showing hyalinization and fibrosis all over the valve $(\mathrm{H}-\mathrm{E} \times 20)$. H-E, hematoxylin and eosin. 
disappeared, and the first sterile culture was obtained on the fourth postoperative day. No further growths on blood culture were detected as well. The patient was discharged from the PICU on the fifth postoperative day, and he was transferred to a regular pediatric ward to complete his preoperative antibiotic treatment. The patient completed the treatment in 5 weeks during his stay in the pediatric ward. Penicillin prophylaxis was advocated as the patient had survived a life-threatening infection (aortic valve S. aureus endocarditis) with the "probability" of rheumatic etiology being the cause of damage to the valve. Even after he completed his preoperative antibiotic treatment, we continued the treatment of prophylaxis with IM benzathine penicillin. The last TTE revealed no mitral regurgitation and normal prostatic valve function. He was discharged from the hospital on the seventh postoperative week. The patient continues to be seen at our pediatric cardiology clinic and is still under treatment for prophylaxis with IM benzathine penicillin 1.2 million units on 21-day basis. Every single echocardiographic examination after he was discharged from the hospital revealed standard prosthetic valve function without displacement.

\section{Discussion}

The conventional treatment for native valve endocarditis due to MSSA consists of antistaphylococcal penicillin, either nafcillin or oxacillin. Cefazolin is an acceptable alternative if there is no central nervous system complication. ${ }^{3}$ Cefotaxime is recommended in nonsevere penicillin allergy by the European Society of Cardiology. ${ }^{4}$ Daptomycin is an acceptable alternative in adults. Fowler et al compared daptomycin use in 124 patients with S. aureus bacteremia and/or endocarditis to standard therapy (gentamicin plus antistaphylococcal penicillin or vancomycin) in 122 patients. Treatment success was similar in two groups ( 44.2 vs. $41.7 \%$ ). In both groups, left-sided IE (LIE) showed low success rates. ${ }^{5}$ However, in a recent multicenter study evaluating 6,075 patients with IE, daptomycin was also beneficial in the treatment of LIE primarily when higher doses $(\geq 8 \mathrm{mg} / \mathrm{kg} / \mathrm{d}$, maximum $12 \mathrm{mg} / \mathrm{kg} / \mathrm{d}$ ) were used. ${ }^{6}$ Similar to these cases, we reported a 15 -year-old pediatric case of Williams' syndrome presenting with $S$. aureus endocarditis, who did not respond to conventional therapy. ${ }^{7}$ Only daptomycin was effective in convalescing the pediatric patient, even though daptomycin is not routinely recommended for use in patients younger than 18 years. In our case, we opted not to use conventional therapy. Instead, daptomycin of $8 \mathrm{mg} / \mathrm{kg} / \mathrm{d}$ was promptly initiated to the patient after obtaining two sets of blood cultures from two different veins.

Daptomycin is a cyclic lipopeptide that is approved by the FDA for treatment of patients $>1$ year of age with complicated skin and skin structure infections (cSSTI) and $>18$ years of age with bacteremia with or without endocarditis due to $S$. aureus. It binds to the bacterial cell membrane in a calcium-dependent manner, causing rapid membrane depolarization and bacterial death. Daptomycin can be an alternative in children with serious infections such as right-sided endocarditis, infection of intravascular devices, and osteoarticular infections when standard antimicrobial therapy fails. In a retrospective report, among 16 children with severe infections due to gram-positive bacteria, the addition of daptomycin resulted in a bacteriologic cure in $85 \%$ of patients with persistent bacteremia. ${ }^{8}$ Infectious Diseases Society of America guidelines on the management of MRSA infections suggest a daptomycin dosage from 6 to 10 $\mathrm{mg} / \mathrm{kg} / \mathrm{d}$ for children with bacteremia, osteomyelitis, and septic arthritis. Daptomycin was used in a dosage of 8 $\mathrm{mg} / \mathrm{kg} / \mathrm{d}$ in our case. Daptomycin is generally well tolerated. As its most relevant side effect is muscular toxicity, regular monitoring of CK levels is recommended. Daptomycin is safe and well tolerated in children with complicated skin infections. ${ }^{9}$ In a retrospective study on 46 children (sepsis, osteomyelitis, cSSTIs, and endocarditis), the overall success rate was 81.2\%. ${ }^{10}$ No increases in CK levels or serum creatinine were observed. Similarly, we did not observe any adverse effects, such as an increase in CK levels or serum creatinine during the treatment of our case.

Referral for early surgery is indicated in LIE in case of valve dysfunction causing symptoms or signs of heart failure; paravalvular extension of infection with development of annular or aortic abscess, destructive penetrating lesion, and/or heart block; and persistent infection, septic embolization, and cerebral emboli. ${ }^{11,12}$ The main reasons for the early surgical intervention in our case were as follows: the extreme deterioration of the cerebral condition of the patient, the rapid spread of septic emboli throughout the patient's extremities and his brain, and the existing of left heart IE. Karaci et al reported successful surgical treatment of IE in seven patients with CHD. Indications for operation included cardiac failure due to atrioventricular septal rupture, severe tricuspid valve insufficiency, and septic embolization in one, moderate valvular dysfunction with vegetations in three (two tricuspid, one mitral), and severe valvular dysfunction with vegetations in the other three patients (two tricuspid, one mitral). ${ }^{11}$ In a 29-year study period by Hickey et al among 30 children, valve replacement was performed in $24 \%$. Univariate predictors for valve replacement included increased leaflet thickening and the occurrence of septic embolization. ${ }^{12}$

\section{Conclusion}

In this article, we described a case of aortic valve S. aureus endocarditis, which was successfully treated with daptomycin in addition to early surgical therapy in the early phase of infection. We suggest that the immediate start of daptomycin in addition to early surgical therapy is crucial and lifesaving in cases of left-sided septic IE.

\section{Authors' Contributions}

All authors participated in creating content for the manuscript, editing, and provided final approval for submission. No undisclosed authors contributed to the manuscript.

\section{Note}

Permission was granted by the parents and patient to publish the case report. 


\section{Funding}

None.

\section{Conflict of Interest \\ None declared.}

\section{References}

1 Toyoda N, Chikwe J, Itagaki S, Gelijns AC, Adams DH, Egorova NN. Trends in infective endocarditis in California and New York state, 1998-2013. JAMA 2017;317(16):1652-1660

2 Irdem A, Baspinar O, Kervancioglu M, Sahin DA, Kilinc MA. Retrospective evaluation of patients with infective endocarditis [in Turkish]. Pediatr Inf 2012;6:127-132

3 Baddour LM, Wilson WR, Bayer AS, et al; Committee on Rheumatic Fever, Endocarditis, and Kawasaki Disease; Council on Cardiovascular Disease in the Young; Councils on Clinical Cardiology, Stroke, and Cardiovascular Surgery and Anesthesia; American Heart Association; Infectious Diseases Society of America. Infective endocarditis: diagnosis, antimicrobial therapy, and management of complications. Circulation 2005;111(23):e394-e434

4 Habib G, Lancellotti P, Antunes MJ, et al; ESC Scientific Document Group. 2015 ESC guidelines for the management of infective endocarditis: the Task Force for the Management of Infective Endocarditis of the European Society of Cardiology (ESC). Endorsed by: European Association for Cardio-Thoracic Surgery (EACTS), the European Association of Nuclear Medicine (EANM). Eur Heart J 2015;36(44):3075-3128
5 Fowler VG Jr, Boucher HW, Corey GR, et al; S. aureus Endocarditis and Bacteremia Study Group. Daptomycin versus standard therapy for bacteremia and endocarditis caused by Staphylococcus aureus. N Engl J Med 2006;355(07):653-665

6 Guleri A, Utili R, Dohmen P, et al. Daptomycin for the treatment of infective endocarditis: results from European Cubicin( $\left({ }^{\circledR}\right)$ Outcomes Registry and Experience (EU-CORE). Infect Dis Ther 2015;4(03): 283-296

7 Yozgat CY, Uzuner S, Yesilbas O, et al. Williams syndrome presenting with intractable Staphylococcus aureusendocarditis. Indian J Pediatr 2020. Doi: 10.1007/s12098-020-03207-0

8 Ardura MI, Mejías A, Katz KS, Revell P, McCracken GH Jr, Sánchez PJ. Daptomycin therapy for invasive gram-positive bacterial infections in children. Pediatr Infect Dis J 2007;26(12): $1128-1132$

9 Bradley J, Glasser C, Patino H, et al. Daptomycin for complicated skin infections: a randomized trial. Pediatrics 2017;139(03): e20162477

10 Garazzino S, Castagnola E, Di Gangi M, et al; SITIP Daptomycin Study Group. Daptomycin for children in clinical practice experience. Pediatr Infect Dis J 2016;35(06):639-641

11 Karaci AR, Aydemir NA, Harmandar B, et al. Surgical treatment of infective valve endocarditis in children with congenital heart disease. J Card Surg 2012;27(01):93-98

12 Hickey EJ, Jung G, Manlhiot C, et al. Infective endocarditis in children: native valve preservation is frequently possible despite advanced clinical disease. Eur J Cardiothorac Surg 2009;35(01): $130-135$ 